Check for updates

Cite this: RSC Adv., 2018, 8, 34839

Received 27th August 2018

Accepted 3rd October 2018

DOI: 10.1039/c8ra07158f

rsc.li/rsc-advances

\section{Biomimetic colloidal photonic crystals by coassembly of polystyrene nanoparticles and graphene quantum dots $\dagger$}

\author{
Dali Huang, $\ddagger^{\mathrm{a}}$ Minxiang Zeng, $\dot{t}^{\mathrm{b}}$ Ling Wang, $\dot{\hbar}^{\mathrm{b}}$ Lecheng Zhang ${ }^{\mathrm{b}}$ \\ and Zhengdong Cheng (ID *ab
}

\begin{abstract}
Biomimetic nanostructured materials with iridescent structural colors have attracted great attention due to their potential in photonic devices, materials science, and biomedical engineering. The technological applications of artificial photonic crystals (PCs), however, are often hindered by their low color visibility. Herein, we report colloidal PCs with enhanced color visibility through the coassembly of thioglycerolmodified graphene quantum dots (GQDs) into the close-packed array of polystyrene (PS) nanospheres. The enhanced polystyrene PCs were fabricated by both centrifugal sedimentation and drop-casting methods. The color visibility of the resulting PCs was found to be strongly dependent on the hydrothermal time (i.e., carbonization) and the doping concentrations of GQDs. The PCs with brilliant reflection colors with red, green and blue (RGB) regions have been achieved by controlling the size of the constituent PS nanoparticles. As a proof of concept for photonic ink applications, we demonstrated a number of photonic images with RGB colors on multiple substrates including paper, silicon wafer and glass. This work is expected to provide new insight into the development of emerging advanced photonic crystals with high color visibility for applications such as colloidal paints, textile fabrics, and wearable displays.
\end{abstract}

\section{Introduction}

Biomimicking the structural iridescent colors of living organisms such as butterfly wings, which may provide an important channel for mate signaling and communication as well as survival in hostile environments, is currently in the research limelight due to their fundamental importance and potential technological applications. ${ }^{1-6}$ It has been demonstrated that the fascinating structural coloration in the wings of morpho butterflies arises from the combination of highly ordered or quasi-amorphous structures and embedded black melanin, ${ }^{7-10}$ with the periodic lattice structures generating the iridescent structural colors while the melanin enhances the color visibility by serving as a background absorber. ${ }^{11,12} \mathrm{~A}$ variety of colloidal photonic crystals have been fabricated through facile self-assembly of different colloidal nanoparticles, such as silica, polystyrene, and poly(methyl methacrylate) into a close-packed array. ${ }^{13-16}$

\footnotetext{
${ }^{a}$ Department of Materials Science \& Engineering, Texas A\&M University, College Station, Texas, 77843, USA. E-mail: zcheng@tamu.edu; Tel: +19798453413

${ }^{b}$ Artie McFerrin Department of Chemical Engineering, Texas A\&M University, College Station, Texas, 77843, USA

$\dagger$ Electronic supplementary information (ESI) available. See DOI: 10.1039/c8ra07158f

$\ddagger$ These authors contributed equally to this work.
}

Among these, colloidal polystyrene nanoparticles are one of the most prominent candidates for fabrication of photonic crystals due to their low expense and relatively simple processing method. Colloidal polystyrene photonic crystals have found diverse applications in areas of displays, ${ }^{17,18}$ paints, ${ }^{19,20}$ and fabrics; ${ }^{21,22}$ however, their low color visibility originated from incoherent scattering remains a hindrance to practical applications. ${ }^{23}$ Inspired by butterfly wings, artificial photonic crystals with enhanced colors have been reported by incorporating various black additives like dark color polymers ${ }^{24,25}$ and magnetic nanoparticles ${ }^{26,27}$ into ordered structures, leading to enhanced color visibility. In addition to synthetic materials, natural biopolymers such as cuttlefish ink $^{28}$ have also demonstrated strong capacity for reducing incoherent light scattering, thus enhancing the color visibility of photonic crystals. With the development of the additives in biomimetic photonic crystals, it also brings new problems. Some undesired defects or dislocations, such as impure phases and missing local particles, however, are often inevitably introduced into the colloidal PCs due to the nonuniform size of these additives and low compatibility with colloidal nanoparticles.

Graphene quantum dots (GQDs) are single- to few-layered graphene nanoparticles with lateral dimensions smaller than $20 \mathrm{~nm}$, which have attracted much research interest due to their unprecedented advantages of unique electronic and 
optical properties, controllable synthesis, cost effectiveness, superior compatibility and high photoluminescent quantum yield. ${ }^{29-42}$ Different from traditional enhanced photonic crystals' research, graphene quantum dots have competitive advantages on working as additives of photonic crystals. First, the morphology of GQDs can be well-tuned and prepared in the nanoscale range $(5-15 \mathrm{~nm})$ such that the small size of GQDs will retain the integrity of PCs, preserving their periodic lattice structures. Second, the surface carbonization of GQDs can be readily modulated by controlling the kinetics of hydrothermal reaction to actively tailor the blackness of GQD additives. Some successful examples of controlled surface carbonization by hydrothermal treatment have been reported for applications like cell labeling, ${ }^{43,44}$ photovoltaic devices ${ }^{45,46}$ and sensors. ${ }^{4,48}$ Last but not least, the chemical stability of GQDs allows the application of photonic arts without significant degradation or oxidation for a long period, which was not easily achievable for biopolymer additives such as cuttlefish inks. These characteristics of GQD additives may successfully start a new direction for building up enhanced photonic crystals.

In this work, colloidal PCs with enhanced color visibility were achieved by coassembly of polystyrene nanoparticles and thioglycerol-modified GQDs. The color visibility of resulting PCs was closely related to the carbonization and the doping concentrations of GQDs. Furthermore, enhanced colloidal PCs with brilliant structural colors covering the full visible spectral range were tuned using PS nanoparticles with different sizes. The resulting colloidal PCs were further used as photonic inks, and such photonic writings/drawings with RGB colors were demonstrated on diverse substrates including paper, glass, and silicon wafer.

\section{Results and discussion}

Fig. 1 represented the synthetic route and characterizations of GQDs. In Fig. 1a, GQDs were fabricated using a controlled hydrothermal reaction of low molecular organic compounds including citric acid, glycine, and 1-thioglycerol. During the $200{ }^{\circ} \mathrm{C}$ pyrolysis process, citric acid and glycine were utilized as main carbon sources and 1-thioglycerol worked as the reducing agent. GQDs with various hydrothermal carbonization degrees were gradually formed due to reaction time difference. The synthesized GQDs were described as GQDs- $x$, in which $x$ was the hydrothermal reaction time of GQDs. Herein, 3 hours, 5 hours, and 7 hours of hydrothermal pyrolysis of GQDs would be referred to as GQDs-3, GQDs-5 and GQDs-7, respectively. The morphology of GQDs-7 was characterized through TEM image, as shown in Fig. 1b. The GQDs-7 had a narrowly distributed size range and the average size was $12.0 \mathrm{~nm}$. The size uniformity of GQDs was also confirmed through AFM image (Fig. S1 $\dagger$ ). Other GQDs TEM images were shown in Fig. S2†. Then Fig. 1c showed the absorption spectra of various GQDs in aqueous suspension. As shown in the figure, the light intensity absorption order of GQDs was GQDs-7 > GQDs-5 > GQDs-3 under the same concentration, $0.2 \mathrm{mg} \mathrm{ml}^{-1}$. This observation confirmed that higher carbonized GQDs with additives could absorb more extra light and would be able to enhance photonic crystals. The absorption intensity of dried GQDs was further characterized by UV-vis spectroscopy in Fig. 1d. The spectra showed high absorption of GQDs in the visible spectrum. The inset image was the photograph of dried GQDs-7.

The reasonable hypothesis was made that the chemical state of GQDs would also change with the hydrothermal processing. Fig. 2 exhibited the X-ray photoelectron spectroscopy (XPS) of (a)

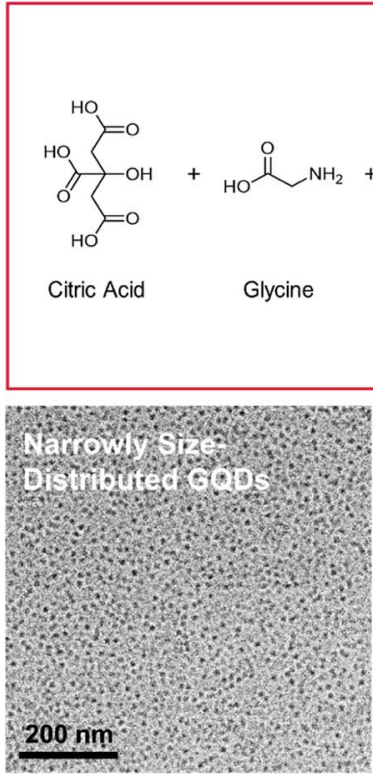

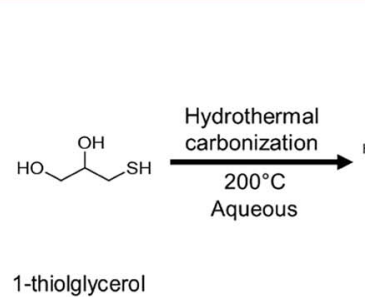

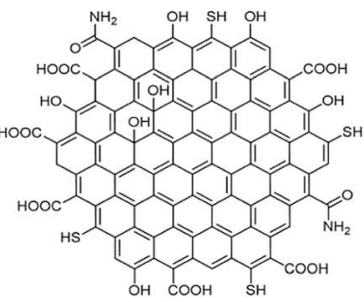

Graphene Quantum Dots
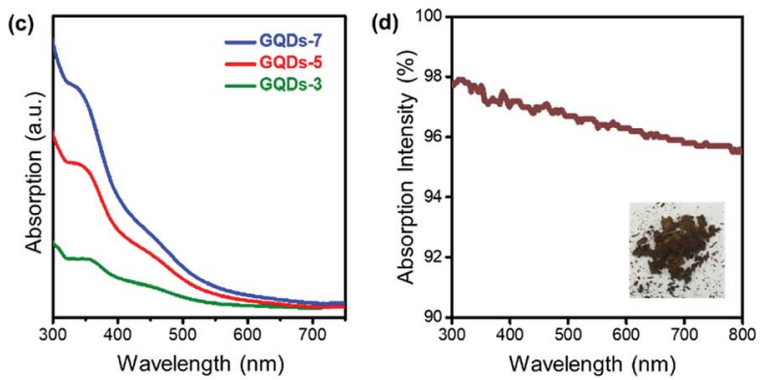

Fig. 1 Fabrication of GQDs. (a) Schematic illustration of the synthesis process of GQDs. (b) TEM images of GQDs-7. (c) Absorption spectra of GQDs aqueous suspension with different reaction time (3 hours, 5 hours, and 7 hours). (d) UV-vis absorption spectra of pure GQDs-7. The inset was the photograph of dried GQDs-7. 
(a)

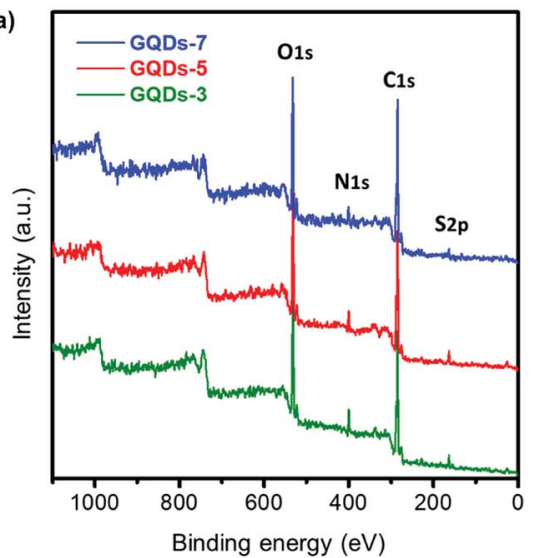

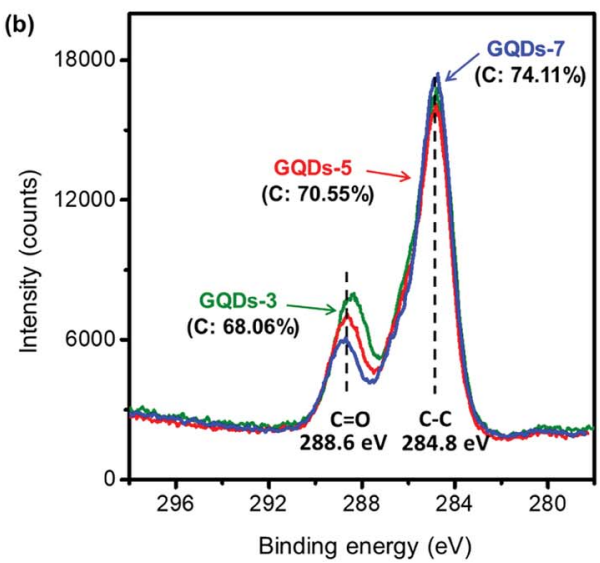

Fig. 2 X-ray photoelectron spectroscopy of GQDs. (a) XPS survey scan of GQDs with reaction time of 3 hours, 5 hours, and 7 hours at $200{ }^{\circ} \mathrm{C}$. (b) XPS carbon chemical state of GQDs with reaction time of 3 hours, 5 hours, and 7 hours. (The $C-C / C=O$ ratios with various reaction times, respectively, were $2.05,2.32$, and 2.95 ).

GQDs. From the XPS survey scan in Fig. 2a, we observed that the synthesized GQDs consisted mainly of four elements: carbon, nitrogen, oxygen, and sulfur. Specifically, detailed change of four elements concentration could be investigated in XPS elemental analysis, as shown in Table 1 . The carbon concentration of GQDs-3, GQDs-5 and GQDs-7 were 68.06\%, 70.55\% and $74.11 \%$, revealing a clear increasing trend of carbonization degree by increasing reaction time. Carbon content increased with GQDs' reaction time, while a consistent loss of nitrogen, oxygen, and sulfur content was observed. To further study the carbonization degree of GQDs, the carbon spectra of XPS under various reaction hours were performed as shown in Fig. $2 b$. The binding energy of $\mathrm{C}-\mathrm{C}$ was $284.8 \mathrm{eV}$ and of which $\mathrm{C}=\mathrm{O}$ was $288.6 \mathrm{eV}$. The peak intensities of $\mathrm{C}-\mathrm{C}, \mathrm{C}=\mathrm{O}$ were obtained from Fig. $2 \mathrm{~b}$ and $\mathrm{C}-\mathrm{C} / \mathrm{C}=\mathrm{O}$ ratio were calculated (data shown in Table $\mathrm{S} 1 \dagger)$. The $\mathrm{C}-\mathrm{C} / \mathrm{C}=\mathrm{O}$ ratio indicated the chemical environment of carbon element as well as the amount of oxygen defects existing in conjugate carbon network, which dictated the absorption capability of visible light, i.e., blackness of GQDs. ${ }^{39}$ The $\mathrm{C}-\mathrm{C} / \mathrm{C}=\mathrm{O}$ ratios were $2.05,2.32$ and 2.95 under the reaction time of 3 hours, 5 hours, and 7 hours, respectively, suggesting that increment of hydrothermal reaction time of GQDs would result in a higher degree of carbonization.

The enhanced PS photonic crystals with various GQDs' reaction time were studied in Fig. 3. Two different methods of fabricating enhanced photonic crystals, centrifugal sedimentation and drop-casting, were introduced in Fig. 3a and b. PS photonic crystals with GQDs would be defined as PS/GQDs, and PS/GQDs- $x$ would be precedingly described as the PS photonic crystals with GQDs which underwent $x$ hours hydrothermal

Table 1 XPS elemental analysis of GQDs with different reaction time

\begin{tabular}{llllll}
\hline & $\mathrm{C} \%$ & $\mathrm{~N} \%$ & $\mathrm{O} \%$ & $\mathrm{~S} \%$ & Total\% \\
\hline GQDs-3 & 68.06 & 5.05 & 23.57 & 3.32 & 100 \\
GQDs-5 & 70.55 & 3.15 & 23.06 & 3.24 & 100 \\
GQDs-7 & 74.11 & 2.48 & 20.26 & 3.16 & 100
\end{tabular}

reaction. Photographs of photonic crystal flakes of PS and enhanced PS/GQDs, taken under natural light, were presented in Fig. 3a. All PS nanoparticles in Fig. 3 were uniform of $214 \mathrm{~nm}$ average size, and GQDs concentration was $1 \mathrm{wt} \%$. From Fig. 3a, pure PS photonic crystals looked pale and barely showed structural color. With increasing hydrothermal reaction time, from 0 to 3 hours, 5 hours, and 7 hours, the crystal flakes progressed to exhibit better green structural color. The size of the enhanced PCs flakes was measured as 1-2 cm. Fig. 3b introduced another drop-casting method of fabricating PS and PS/ GQDs photonic crystals films. The films were prepared through using a pipette to drop casting a fixed amount of PS/ GQDs suspension in water onto the black paper. The paper was heated on the $50{ }^{\circ} \mathrm{C}$ hot surface resulting in PCs films with a flatter surface compared with those crystal flakes due to the quick self-assembling process. From Fig. 3b, PCs films were differentiated by the GQDs' hydrothermal reaction time. Under the same $1 \mathrm{wt} \%$ GQDs concentration, the color visibility of PS/ GQDs photonic crystal films was better than that of the pale PS film. The PS/GQDs-7 photonic crystals films had the best color visibility, progressing to lesser color visibility accompanied with shorter hydrothermal processing time.

The reflectance spectra of photonic crystals films, which confirmed their visual appearance, were investigated in Fig. 3c. The reflectance spectra of pure PS thin films had the largest reflectance intensity of all. High reflectance intensity would come with poor color visibility. The structural color of pure PS photonic crystals appeared milky white and pale, being strongly suppressed by scattering and background light. By tuning hydrothermal reaction time of GQDs additives (3, 5 and 7 hours), the carbonization of GQDs additives was controlled simply, achieving the desired small reflectance intensity of photonic crystals films. Longer GQDs hydrothermal pyrolysis time resulted in larger carbonization, with PS/GQDs photonic crystals films having accordingly smaller reflectance intensity and higher color visibility. Fig. 3d showed the corresponding normalized reflectance peak intensity of Fig. 3c (the normalized reflectance spectra were presented in Fig. S3†). PS/GQDs 
(a)

(b)

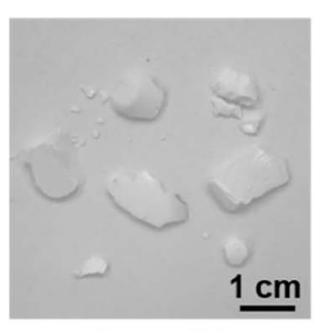

Pure PS

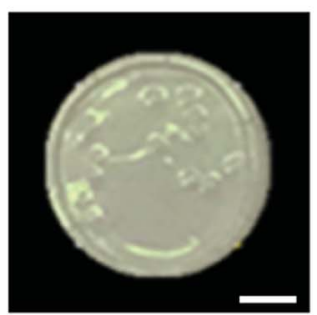

Pure PS

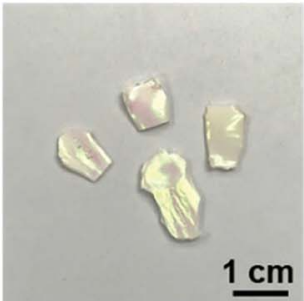

PS/GQDs-3

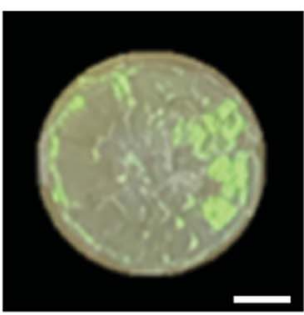

PS/GQDs-3

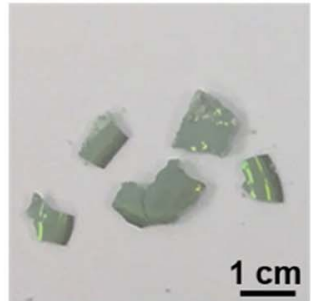

PS/GQDs-5

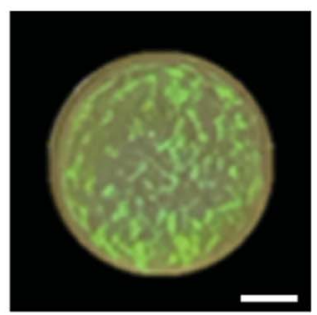

PS/GQDs-5

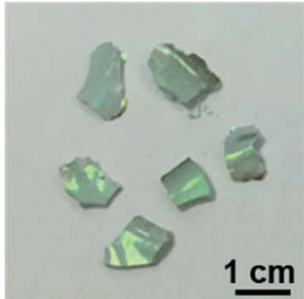

PS/GQDs-7

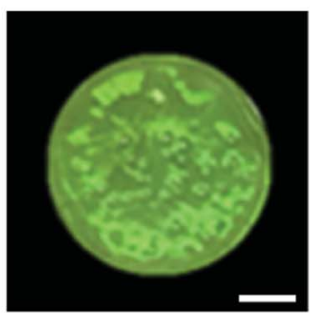

PS/GQDs-7 (c)

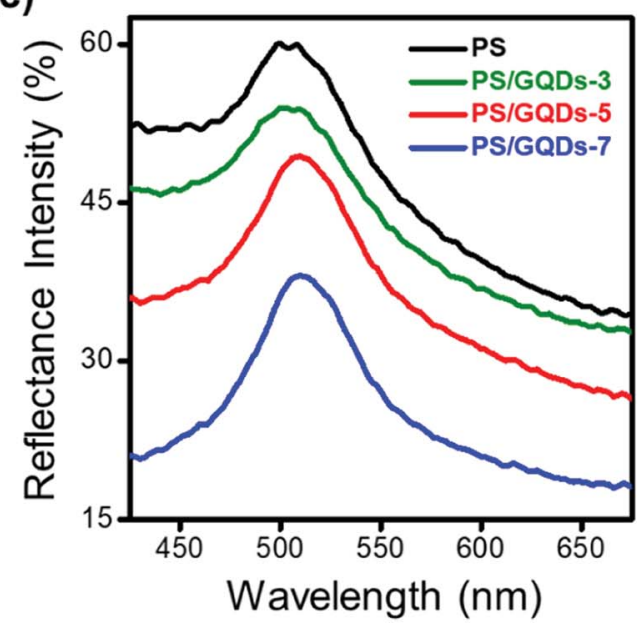

(d)

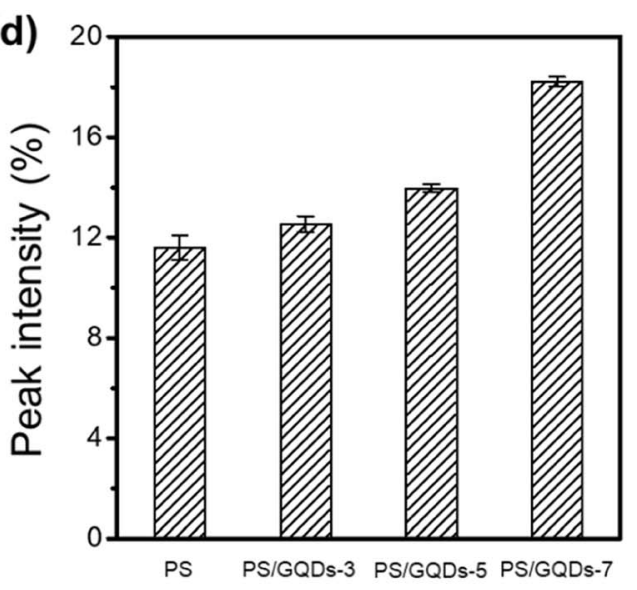

Fig. 3 Fabrication of enhanced PS photonic crystals with different carbonization of GQDs. (a) Photographs of PS/GQDs photonic crystal flakes by centrifugal sedimentation method. GQDs reaction time from left to right was 0,3 hours, 5 hours and 7 hours. (b) Photographs of PS/GQDs photonic crystal films by drop-casting method. The scale bar is $2.5 \mathrm{~mm}$. (c) Reflectance spectra of PS/GQDs photonic crystal films. (d) Corresponding normalized reflectance peak intensity of the PS/GQDs photonic crystals. (The size of PS nanoparticles was $214 \mathrm{~nm}$. The various degrees of carbonization of GQDs were at the same concentration of $1 \mathrm{wt} \%$.)

photonic crystals films with higher carbonized GQDs had larger normalized reflectance peak intensity compared with PS/GQDs photonic crystals of smaller carbonized GQDs and pure PS.

Interestingly, apart from the effect of carbonization of GQDs, the GQDs additives dosage in PS photonic crystals could also affect color visibility. Fig. 4 demonstrated the fabrication of enhanced PS photonic crystals at various GQDS concentrations. The enhanced PS/GQDs photonic crystal films were shown in Fig. 4a. The polystyrene nanoparticle size was $214 \mathrm{~nm}$, and the GQDs had undergone hydrothermal reaction of 7 hours. The PC films were also fabricated by the dropcasting method, with all films having around $1 \mathrm{~cm}$ diameter. From visual perception, it was apparent that higher GQDs doping concentration in PCs films created more brilliant green structural color. The reflectance spectra shown in Fig. 4b further clarified the reflectance mechanism of enhanced photonic crystals. Pure PS thin films showed relatively high reflectance intensity. When GQDs-7 (0.1 wt $\%, 0.5 \mathrm{wt} \%$, and $1 \mathrm{wt} \%$ ) were added to the PS photonic crystals, the visible reflectance intensity started to decrease. Larger GQDs additives concentration resulted in lower reflectance intensity of photonic crystals. This might result from the incoherent light absorption by the GQDs. Higher concentration of GQDs inside PS photonic crystals would absorb more background and scattering light, while enhanced photonic crystals exhibited better color visual appearance. The reflectance peak intensity of PS/GQDs photonic crystals in Fig. $4 \mathrm{~b}$ was normalized, as shown in Fig. 4c (the normalized spectra were shown in Fig. S4†). With the GQDs-7 concentration increasing from $0 \mathrm{wt} \%$ to $1 \mathrm{wt} \%$, the normalized peak intensity increased from $11.58 \%$ to $18.22 \%$, which implied the peak intensity increment of GQDs addition. 
(a)
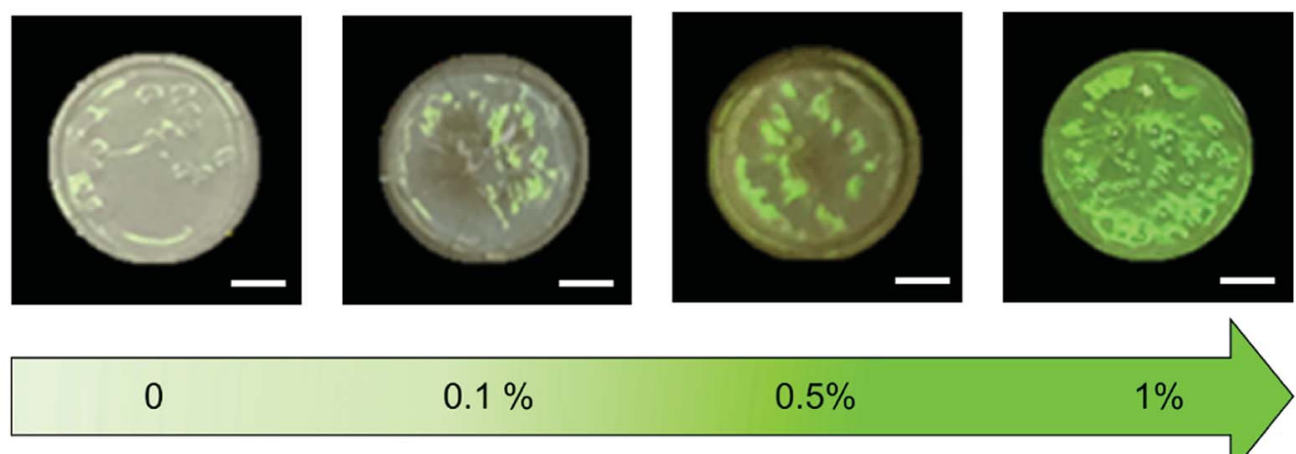

(b)

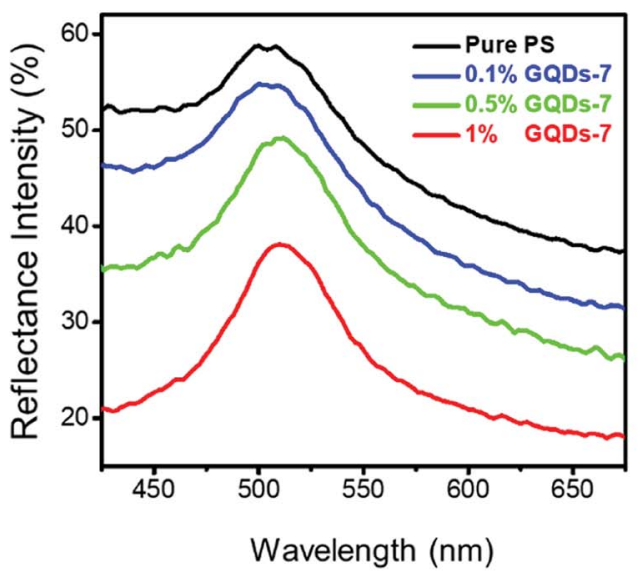

(c)

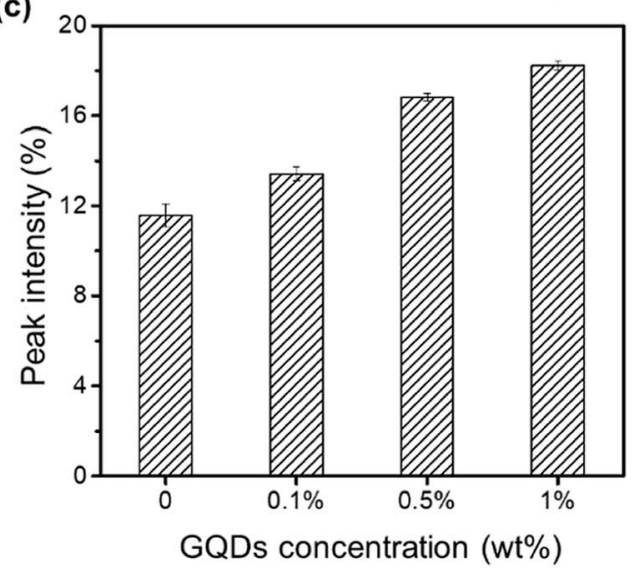

Fig. 4 Enhanced PS photonic crystals with various GQDs concentrations. (a) Photographs of polystyrene PCs films at various GQDs concentration. The concentration from left to right, was $0 \mathrm{wt} \%, 0.1 \mathrm{wt} \%, 0.5 \mathrm{wt} \%, 1 \mathrm{wt} \%$. The scale bar is $2.5 \mathrm{~mm}$. (b) Reflectance spectra of PS/GQDs photonic crystal films. (c) Corresponding normalized reflection peak intensity of PS/GQDs photonic crystal films. (The size of PS nanoparticles was $214 \mathrm{~nm}$. GQDs underwent hydrothermal reaction for 7 hours.)

After green color PCs enhancement, full visible spectra range color enhancement PCs was also achieved through tuning the particle size of PCs and the concentration of GQDs. The enhanced coloring of PS nanoparticles with GQDs was shown in Fig. 5. In addition to green color group PCs, blue, red and cyan enhanced PCs with GQDs (Fig. 5a) were also fabricated by dropcasting method. Four different sizes of uniform PS nanoparticles were synthesized and the diameters were measured as 192, 214, 274 and $396 \mathrm{~nm}$ from both SEM and DLS measurement (Fig. S5 and S6†). PS nanoparticles also went through the zeta potential test (Table S3†). The corresponding PCs films were aligned in four rows showing blue, green, red and cyan structural color groups. Each row in Fig. 5a represented a color group PS/GQDs photonic crystal films with variable GQDs additive concentration. The GQDs concentration was $0 \mathrm{wt} \%$, $0.1 \mathrm{wt} \%, 0.25 \mathrm{wt} \%, 0.5 \mathrm{wt} \%$ and $1 \mathrm{wt} \%$. The color visibility also increased each row from left to right, with the leftmost palest and the rightmost representing best brilliant structural color.

The reflectance peaks of four PS/GQDs films conducted through UV-vis spectrofluorometer was $446 \mathrm{~nm}, 510 \mathrm{~nm}$, $640 \mathrm{~nm}$, and $472 \mathrm{~nm}$, as shown in Fig. 5b. The PS/GQD films compositions were PS nanoparticles (192, 214, 274 and $396 \mathrm{~nm})$ with 1 wt $\%$ GQDs-7. The peaks data achieved could be compared with theoretical calculations. For the visible wavelength range photonic crystals, the reflectance mechanism of photonic crystals could be explained through Bragg's law. The reflectance wavelength of light $\lambda$ could be represented by eqn (1):

$$
\lambda=\frac{1}{K} 2 \sqrt{\frac{2}{3}} D_{\mathrm{c}-\mathrm{c}} \sqrt{n_{\text {effective }}^{2}-\sin ^{2} \theta}
$$

where $\lambda$ is the reflectance wavelength of light, $K$ is the order of reflection, $D_{\mathrm{c}-\mathrm{c}}$ is the distance between two nearest nanoparticles, $n_{\text {effective }}$ is the effective refractive index of photonic crystal and $\theta$ is the incident angle. Accordingly, in PS/GQDs enhanced photonic crystals composites, $n_{\text {effective }}$ would consist of the refractive index of the PS nanoparticles, the PS interstitial space air and GQDs. The effective refractive index could be defined by eqn (2):

$$
n_{\text {effective }}=\emptyset_{\mathrm{PS}} n_{\mathrm{PS}}+\varnothing_{\mathrm{Air}} n_{\mathrm{Air}}+\varnothing_{\mathrm{GQDs}} n_{\mathrm{GQDs}}
$$

where $n_{\mathrm{PS}}, n_{\mathrm{Air}}$ and $n_{\mathrm{GQDS}}$ represented refractive index of PS $(n=$ 1.59), air $(n=1)$ and GQDs $(n=1.88)$, respectively. $\varnothing_{\mathrm{PS}}, \varnothing_{\mathrm{air}}$ and $\emptyset_{\mathrm{GQDs}}$ represented the volume fraction of PS $(\varnothing=74 \%)$, air $(\varnothing=25 \%)$ and GQDs $(\varnothing=1 \%) . n_{\text {effective was herein calculated as }}$ 1.4454. To calculate reflectance wavelength $\lambda$ of PS/GQDs photonic crystals, $D_{\mathrm{c}-\mathrm{c}}$ was considered equal to the size of the 
(a)

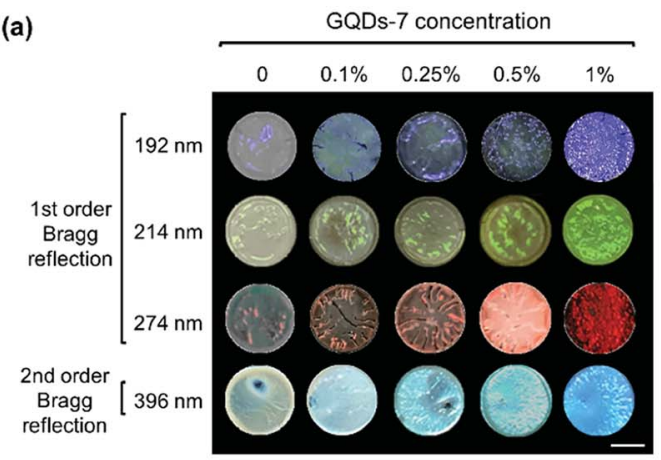

(c)

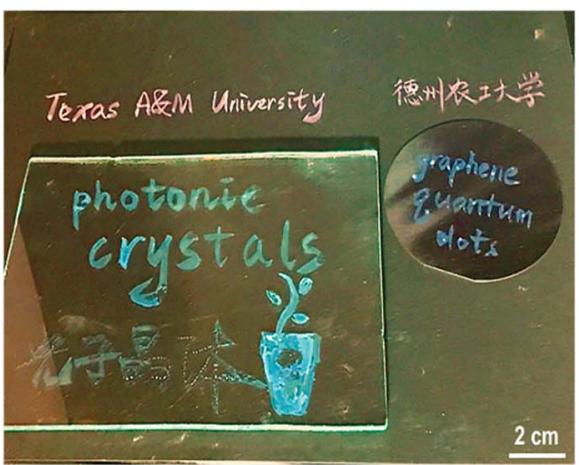

(b)

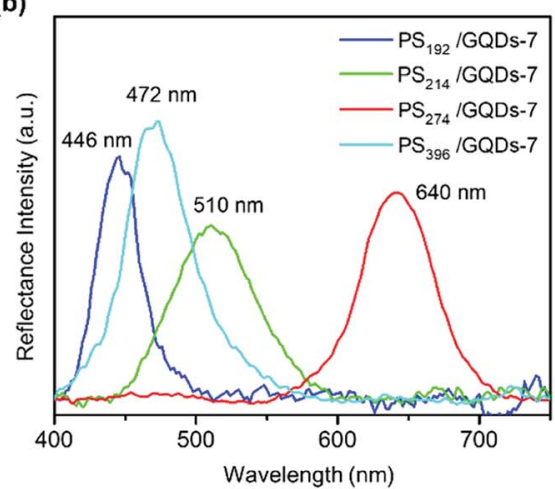

(d)

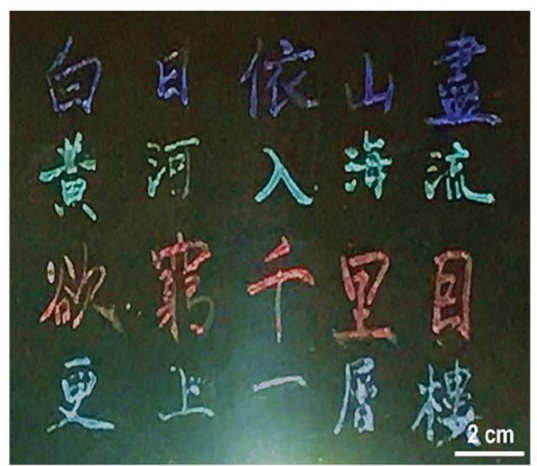

Fig. 5 Coloring of PS nanoparticles with GQDs. (a) Photographs of PS nanoparticles (192, 214, 274, and 396 nm) at various GQDs-7 weight concentration. Concentration of GQDs additive increases from left to right. The scale bar is $5 \mathrm{~mm}$. (b) Reflectance spectra of PS/GQDs thin film. The PS nanoparticles sizes are 192, 214, 274, and $396 \mathrm{~nm}$. The GQDs-7 concentration is $1 \mathrm{wt} \%$. (c) Photograph of photonic ink writings on different substrates (paper, glass and wafer). (d) Photograph of photonic ink calligraphy of a Chinese Tang dynasty famous poem "On the Stork Tower".

average PS nanoparticle (192, 214, 274, and $396 \mathrm{~nm})$ due to close packed FCC structure. The incident angle $\theta$ was $0^{\circ}$ because the incident angle was perpendicular to the surface. The refractive index $n_{\text {effective }}$ was 1.4454, and order of Bragg reflection $K$ is integer 1 or 2 . Detailed calculations could be seen in Table S2. $\dagger$ The calculated $\lambda$ of PS/GQDs films (PS sizes: 192, 214, 274, and $396 \mathrm{~nm}$ ) were $453.18 \mathrm{~nm}, 505.11 \mathrm{~nm}, 646.73 \mathrm{~nm}$, and
$467.35 \mathrm{~nm}$, respectively. The calculated peaks closely matched with experimental test wavelengths and proved their credibility after comparison. From the simulated calculation of Bragg's law and UV-vis characterization, it was found that the colors of blue, green, and red thin films resulted from the 1st order of Bragg reflection, and cyan color thin film resulted from the 2nd order of Bragg reflection.

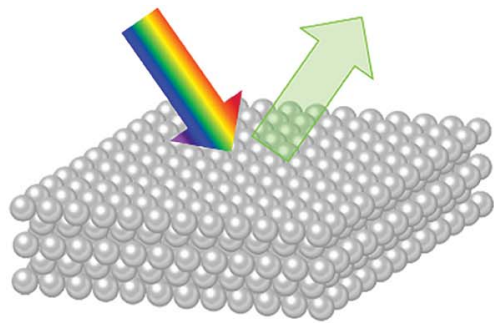

Colloidal photonic crystal

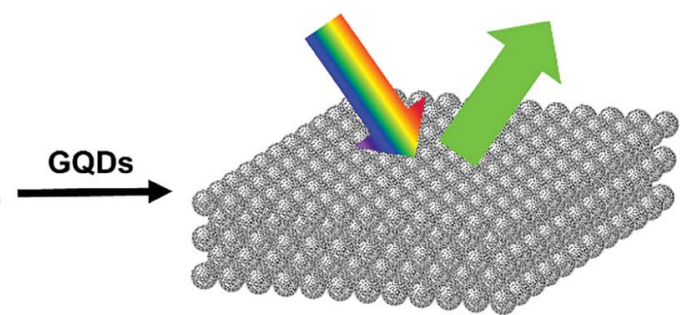

Enhanced colloidal photonic crystal

Fig. 6 Schematic illustration of the Bragg-reflection color enhancement of PS and PS/GQDs colloidal photonic crystals. The size of PS nanoparticle is $214 \mathrm{~nm}$. 
The PS/GQDs enhanced photonic crystals with various structural colors could be directly utilized as photonic inks for painting or calligraphy. Fig. $5 \mathrm{c}$ represented photonic ink writings and drawings on diverse substrates like paper, silicon wafer and glass. And Fig. 5d showed the photograph of photonic ink Chinese calligraphy, which was a Chinese Tang dynasty eternally famous poem "On the Stork Tower" by poet Zhihuan Wang. The results exhibited the great potential of enhanced photonic crystals in painting and printing areas. One ink brush was used for all writings and drawings in Fig. 5c and d.

The possible mechanism of Bragg reflection color enhancement of colloidal photonic crystals is schematically illustrated in Fig. 6. The synthetic photonic crystal presented structural colors under natural daylight. Monodisperse PS nanoparticles $(214 \mathrm{~nm})$ with ordered Bravais lattice structures formed the colloidal photonic crystals with comparatively pale green structural color. The small GQDs tended to aggregate around the big PS nanoparticles as verified by TEM characterization (Fig. S7a $\uparrow$ ), as a result, GQDs entered the void space of colloidal photonic crystals and coassembled to the surface of PS during the drying process. It should be noted that the lattice structure of photonic crystal did not show obvious changes with the addition of a slight amount of GQDs as shown in SEM images (Fig. S7b and S7c $\dagger$ ). From Table S3, $\uparrow$ the PS nanoparticles were negatively charged and GQDs were positively charged. Their electron polarity difference would also speed up the coassembly of PS and GQDs during the drying process. The GQDs localized in the interstitial space reduced the incoherent light scattering, therefore brilliant structural colors with high visibility were observed in such colloidal photonic crystals.

\section{Conclusions}

In conclusion, colloidal photonic crystals with enhanced color visibility was accomplished through the coassembly of PS nanospheres with thioglycerol-modified GQDs. For the first time, the GQDs additives were found to exhibit superior compatibility with PS nanoparticles and could reduce incoherent scattering and background light of resulting colloidal PCs. High color visibility of colloidal PCs could be achieved by increasing the doping concentration of thioglycerol-modified GQDs with a high degree of carbonization. We also demonstrated colloidal PCs with enhanced color visibility in a fullvisible-wavelength range by tuning the size of PS nanoparticles. Brilliant structural colors of enhanced photonic crystals could be used as photonic ink for art writings and drawings. The results disclosed here are expected to pave an attractive avenue for developing multifunctional colloidal photonic crystals with high color visibility towards their applications in areas of textile fabrics and wearable information display, as well as colloidal ink and paints.

\section{Experimental}

\subsection{Reagents}

Chemicals were purchased from commercial suppliers and were used without further purification. For preparation of
GQDs, citric acid (purity > 99\%), 1-thioglycerol (purity $\geq 97 \%$ ), and glycine (purity $\geq 98.5 \%$ ) were purchased from SigmaAldrich. For preparation of PS nanoparticles, styrene (stabilized, purity $>99 \%$ ) was purchased from Acros Organics. Methyl methacrylate was obtained from Polysciences, Inc. Acrylic acid (purity > 99\%), ammonium persulfate (purity $\geq 99.99 \%$ ), sodium dodecylbenzenesulfonate (technical grade), and sodium bicarbonate (purity $\geq 99.7 \%$ ) were purchased from Sigma-Aldrich.

\subsection{Experiments}

4.2.1 Synthesis of thioglycerol-modified GQDs. Thioglycerol-modified GQDs were prepared using the traditional hydrothermal pyrolysis method. $2.40 \mathrm{~g}$ citric acid and $1.0 \mathrm{~g}$ glycine were dissolved in $5 \mathrm{ml}$ deionized water, then $1.07 \mathrm{~g}$ 1-thioglycerol was added, after that sonicated for 15 minutes. The solution was then decanted into an $80 \mathrm{ml}$ Teflon vessel and sealed within a stainless autoclave reactor. The autoclaves were heated to $200{ }^{\circ} \mathrm{C}$ and three different reactions were prepared with 3 hours, 5 hours and 7 hours, separately. The reaction products were centrifuged at $10000 \mathrm{rpm}$ for 15 minutes to remove solid residue.

4.2.2 Preparation of colloidal PS nanoparticles. Monodisperse PS nanoparticles were fabricated using the emulsion polymerization method. In a $500 \mathrm{ml}$ bottom-boiling flask, $28.0 \mathrm{~g}$ styrene, $1.1 \mathrm{~g}$ acrylic acid, $1.5 \mathrm{~g}$ methyl methacrylate, and $200 \mathrm{ml}$ deionized water were heated to $85{ }^{\circ} \mathrm{C}$ under magnetic stirring and nitrogen flow. Then $0.725 \mathrm{~g}$ ammonium persulfate (APS), 2$9 \mathrm{mg}$ sodium dodecylbenzenesulfonate (SDBS), and $500 \mathrm{mg}$ $\mathrm{NaHCO}_{3}$ were added into the solution. The PS nanoparticles were achieved after continuous heating at $85^{\circ} \mathrm{C}$ for 7 hours. The size of synthesized PS nanoparticles was primarily tuned by varying the SDBS amount. Monodispersed PS colloidal nanoparticles of different sizes (192, 214, 274 and $396 \mathrm{~nm}$ ) were purified by washing three times with water. The PS suspension was subjected to a freeze-drying process to produce dried monodisperse PS powder.

4.2.3 Fabrication of PCs flakes by the centrifugal sedimentation method. The PCs flakes were prepared by the centrifugal sedimentation method. $200 \mathrm{mg}$ PS powder was added into different volumes of $0.2 \mathrm{mg} \mathrm{ml}^{-1}$ GQDs solution (0 wt $\%, 0.1 \mathrm{wt} \%, 0.5 \mathrm{wt} \%$, and $1 \mathrm{wt} \%$ ). The suspension was transferred to a $50 \mathrm{ml}$ centrifuge tube into which deionized water added to fill to an amount of $35 \mathrm{ml}$. The solution was shaken on a vortex mixer and centrifuged at $10000 \mathrm{rpm}$ for 45 minutes. Then the PCs flakes were taken out by a spoon spatula after removal of solution followed by drying on the $50{ }^{\circ} \mathrm{C}$ hot plate.

4.2.4 Fabrication of PCs films by drop-casting method. The PCs film was prepared by the drop-casting method. The PCs composite suspension was prepared by mixing fixed amounts of PS powder and $0 \mathrm{wt} \%, 0.1 \mathrm{wt} \%, 0.5 \mathrm{wt} \%$, and $1 \mathrm{wt} \%$ GQDs, then deionized water was added to make a solution density of PS nanoparticles of $100 \mathrm{mg} \mathrm{ml} \mathrm{m}^{-1}$. The solution was shaken by vortex mixer and put under sonication for 10 minutes to achieve uniformity. A black paper was placed on the $50{ }^{\circ} \mathrm{C}$ hot plate 
surface. Then $100 \mu \mathrm{l}$ mixed suspension was dropped onto the black paper and allowed to dry. The enhanced PCs thin film was made after complete drying.

\subsection{Characterizations}

The morphologies of nanoparticles and composites were imaged using a Quanta 600 field emission scanning electron microscopy (FESEM) and JEOL 2010 transmission electron microscopy (TEM). SEM samples were sputter coated to $3 \mathrm{~nm}$ thickness Pt/Pd alloy. The size distribution of particles was tested through Bruker Dimension Icon AFM. Raman spectra was tested in Horiba Jobin-Yvon LabRam HR. The absorption and reflectance spectra intensity of both liquid and solid samples were conducted with the Hitachi U-4100 UV-Vis highresolution spectrophotometer. The photoluminescence intensity was measured with the PTI QuantaMaster series spectrofluorometer. The X-ray photoelectron spectroscopy (XPS) spectra of composition and element chemical state were obtained by Omicron's DAR 400 series XPS system with $\mathrm{Mg} \mathrm{K} \alpha$ radiation. The Malvern ZetaSizer NANO NS-90 was used for dynamic light scattering (DLS) testing to measure the average size of PS particles and for zeta potential tests.

\section{Conflicts of interest}

There are no conflicts to declare.

\section{Acknowledgements}

This work is partially financially supported by NASA (NASANNX13AQ60G). The authors are thankful to Dr Mustafa Akbulut's research group for technical support in DLS test. We acknowledge Microscopy and Imaging Center at Texas A\&M University and Materials Characterization Facility at Texas A\&M University for performing AFM, SEM and TEM.

\section{References}

1 P. Vukusic, J. Sambles and C. Lawrence, Nature, 2000, 404, 457.

2 R. H. Siddique, S. Vignolini, C. Bartels, I. Wacker and H. Hölscher, Sci. Rep., 2016, 6, 36204.

3 C. E. Pinheiro, A. V. Freitas, V. C. Campos, P. J. DeVries and C. M. Penz, Neotrop. Entomol., 2016, 45, 107-113.

4 S. Kinoshita and S. Yoshioka, ChemPhysChem, 2005, 6, 14421459.

5 R. A. Potyrailo, H. Ghiradella, A. Vertiatchikh, K. Dovidenko, J. R. Cournoyer and E. Olson, Nat. Photonics, 2007, 1, 123128.

6 L. Wang and Q. Li, Chem. Soc. Rev., 2018, 47, 1044-1097.

7 D. G. Stavenga, H. L. Leertouwer and B. D. Wilts, J. Exp. Biol., 2014, 217, 2171-2180.

8 B. D. Wilts, T. M. Trzeciak, P. Vukusic and D. G. Stavenga, J. Exp. Biol., 2012, 215, 796-805.
9 K. Chung, S. Yu, C. J. Heo, J. W. Shim, S. M. Yang, M. G. Han, H. S. Lee, Y. Jin, S. Y. Lee, N. Park and J. H. Shin, Adv. Mater., 2012, 24, 2375-2379.

10 S. Yoshioka and S. Kinoshita, Proc. Biol. Sci., 2006, 273, 129134.

11 S. Kinoshita, S. Yoshioka and J. Miyazaki, Rep. Prog. Phys., 2008, 71, 076401.

12 M. Xiao, Y. Li, M. C. Allen, D. D. Deheyn, X. Yue, J. Zhao, N. C. Gianneschi, M. D. Shawkey and A. Dhinojwala, ACS Nano, 2015, 9, 5454-5460.

13 J. Xu, W. Li, P. Shen, Y. Li, Y. Li, Y. Deng, Q. Zheng, Y. Liu, Z. Ding, J. Li and T. Zheng, Microchim. Acta, 2017, 184, 3755-3763.

14 G. Liu, L. Zhou, Y. Wu, C. Wang, Q. Fan and J. Shao, J. Appl. Polym. Sci., 2015, 132, 41750.

15 R. De Angelis, I. Venditti, I. Fratoddi, F. De Matteis, P. Prosposito, I. Cacciotti, L. D'Amico, F. Nanni, A. Yadav, M. Casalboni and M. V. Russo, J. Colloid Interface Sci., 2014, 414, 24-32.

16 L. Wang, H. K. Bisoyi, Z. Zheng, K. G. Gutierrez-Cuevas, G. Singh, S. Kumar, T. J. Bunning and Q. Li, Mater. Today, 2017, 20, 230-237.

17 D. Ge, E. Lee, L. Yang, Y. Cho, M. Li, D. S. Gianola and S. Yang, Adv. Mater., 2015, 27, 2489-2495.

18 Z. Cheng, W. B. Russel and P. Chaikin, Nature, 1999, 401, 893.

19 Z. Wu, D. Lee, M. F. Rubner and R. E. Cohen, Small, 2007, 3, 1445-1451.

20 H. Cong, B. Yu, S. Wang, L. Qi, J. Wang and Y. Ma, Opt. Express, 2013, 21, 17831-17838.

21 Y. Li, L. Zhou, G. Zhang, G. Liu, Q. Fan and J. Shao, Surf. Coat. Technol., 2017, 319, 267-276.

22 J. Shao, Y. Zhang, G. Fu, L. Zhou and Q. Fan, J. Text. Inst., 2014, 105, 938-943.

23 X. Yang, D. Ge, G. Wu, Z. Liao and S. Yang, ACS Appl. Mater. Interfaces, 2016, 8, 16289-16295.

24 B. Yi and H. Shen, Chem. Commun., 2017, 53, 9234-9237.

25 D. Ge, X. Yang, Z. Chen, L. Yang, G. Wu, Y. Xia and S. Yang, Nanoscale, 2017, 9, 17357-17363.

26 J. Ge and Y. Yin, J. Mater. Chem., 2008, 18, 5041-5045.

27 J. Ge, J. Goebl, L. He, Z. Lu and Y. Yin, Adv. Mater., 2009, 21, 4259-4264.

28 Y. Zhang, B. Dong, A. Chen, X. Liu, L. Shi and J. Zi, Adv. Mater., 2015, 27, 4719-4724.

29 A. Cao, Z. Liu, S. Chu, M. Wu, Z. Ye, Z. Cai, Y. Chang, S. Wang, Q. Gong and Y. Liu, Adv. Mater., 2010, 22, 103-106.

30 Q. Xiang, F. Cheng and D. Lang, ChemSusChem, 2016, 9, 9961002.

31 W. Cai and X. Chen, Small, 2007, 3, 1840-1854.

32 M. Bacon, S. J. Bradley and T. Nann, Part. Part. Syst. Charact., 2014, 31, 415-428.

33 X. Li, M. Rui, J. Song, Z. Shen and H. Zeng, Adv. Funct. Mater., 2015, 25, 4929-4947.

34 J. Peng, W. Gao, B. K. Gupta, Z. Liu, R. Romero-Aburto, L. Ge, L. Song, L. B. Alemany, X. Zhan, G. Gao, S. A. Vithayathil, B. A. Kaipparettu, A. A. Marti, T. Hayashi, J. J. Zhu and P. M. Ajayan, Nano Lett., 2012, 12, 844-849. 
35 S. Min, J. Hou, Y. Lei, X. Ma and G. Lu, Appl. Surf. Sci., 2017, 396, 1375-1382.

36 J. Ge, M. Lan, B. Zhou, W. Liu, L. Guo, H. Wang, Q. Jia, G. Niu, X. Huang and H. Zhou, Nat. Commun., 2014, 5, 4596. 37 L. Lin and S. Zhang, Chem. Commun., 2012, 48, 10177-10179. 38 X. Miao, D. Qu, D. Yang, B. Nie, Y. Zhao, H. Fan and Z. Sun, Adv. Mater., 2018, 30, 1704740.

39 Y. Dong, J. Shao, C. Chen, H. Li, R. Wang, Y. Chi, X. Lin and G. Chen, Carbon, 2012, 50, 4738-4743.

40 S. Park, N. Mohanty, J. W. Suk, A. Nagaraja, J. An, R. D. Piner, W. Cai, D. R. Dreyer, V. Berry and R. S. Ruoff, Adv. Mater., 2010, 22, 1736-1740.

41 M. Zeng, S. A. Shah, D. Huang, D. Parviz, Y.-H. Yu, X. Wang, M. J. Green and Z. Cheng, ACS Appl. Mater. Interfaces, 2017, 9, 30797-30804.
42 X. Ma, Q. Xiang, Y. Liao, T. Wen and H. Zhang, Appl. Surf. Sci., 2018, 457, 846-855.

43 M. Zhang, L. Bai, W. Shang, W. Xie, H. Ma, Y. Fu, D. Fang, H. Sun, L. Fan and M. Han, J. Mater. Chem., 2012, 22, 7461-7467.

44 W. Shang, X. Zhang, M. Zhang, Z. Fan, Y. Sun, M. Han and L. Fan, Nanoscale, 2014, 6, 5799-5806.

45 V. Gupta, N. Chaudhary, R. Srivastava, G. D. Sharma, R. Bhardwaj and S. Chand, J. Am. Chem. Soc., 2011, 133, 9960-9963.

46 Y. Li, Y. Hu, Y. Zhao, G. Shi, L. Deng, Y. Hou and L. Qu, Adv. Mater., 2011, 23, 776-780.

47 J. Zhao, G. Chen, L. Zhu and G. Li, Electrochem. Commun., 2011, 13, 31-33.

48 Y. Dong, G. Li, N. Zhou, R. Wang, Y. Chi and G. Chen, Anal. Chem., 2012, 84, 8378-8382. 